\title{
RIEMANNIAN FOLIATIONS OF THE RANK ONE SYMMETRIC SPACES
}

\author{
RICHARD H. ESCOBALES, JR.
}

In memory of Professor Yozo Matsushima

\begin{abstract}
In this paper Riemannian folfiations of spheres by spheres and of projective spaces by projective spaces of the same kind are classified by using earlier results of the author and a theorem of Ucci.
\end{abstract}

In this note we classify nontrivial bundle-like or Riemannian foliations of spheres by spheres and nontrivial Riemannian foliations of certain projective spaces by projective spaces of the same kind. The results on projective spaces depend in a crucial way on a theorem of Ucci [11] and some earlier works of the author $[2,3]$. These latter results were discussed at a conference at East Lansing. I wish to thank Professor Philippe Tondeur for the invitation to speak at the special session on foliations (November 13, 1982). Some comments by Professors Larry Conlon and Herman Gluck gave me renewed hope that Riemannian foliations of spheres by great circles could be classified-although the present argument is still somewhat formal. I am grateful to them and to Professor Ucci for his interest. $\$ 1$ recalls some preliminary material, and $\$ 2$ contains the main results and their proofs.

1. Throughout this paper $M$ is assumed to be a $C^{\infty}$ manifold which is connected and complete. Assume $M$ has a codimension $q$ foliation which will be denoted by $\mathscr{V}$. Then this foliation may be defined by a family of $C^{\infty}$ submersions $f_{\alpha}$ : $U_{\alpha} \rightarrow f_{\alpha}\left(U_{\alpha}\right) \subset R^{q}$ where $\left\{U_{\alpha}\right\}_{\alpha \in \Lambda}$ is a maximal open cover of $M$ and where, for each $p \in U_{\alpha} \cap U_{\beta}$, there is a local $C^{\infty}$ diffeomorphism $\phi_{\beta \alpha}^{p}$ of $R^{q}$ so $f_{\beta}=\phi_{\beta \alpha}^{p} \circ f_{\alpha}$ in some neighborhood $U_{p}$ of $p$. For $p^{\prime} \in U_{\alpha} \cap U_{\beta}, \phi_{\beta \alpha}^{p^{\prime}}=\phi_{\beta \alpha}^{p}$ on $f_{\alpha}\left(U_{p} \cap U_{p^{\prime}}\right)$ and $\phi_{\beta \alpha}^{p}=\phi_{\beta \gamma}^{p} \phi_{\gamma \alpha}^{p}$ whenever this makes sense [7]. If we equip $M$ with a Riemannian metric and the open sets $f_{\alpha}\left(U_{\alpha}\right) \subset R^{q}$ with local metrics, then the foliation is Riemannian (or bundle-like) provided each $f_{\alpha}$ is a Riemannian submersion. It should be noted that in general the local metrics are not flat.

Let us agree that a foliation of a manifold $M$ is trivial if either $M$ is foliated by points (in which case the leaf space is $M$ itself) or if $M$ is foliated by itself (in which case the leaf space is a point). In the case of the projective planes $F P(n)$ with $F$ denoting the complex field $C$ or the quaternionic numbers $H, F P(0)$ will be

Received by the editors August 10, 1984 and, in revised form, January 22, 1985.

1980 Mathematics Subject Classification. Primary 57B30; Secondary 53C25. 
regarded as a point. In what follows, we assume that $F P(k)$ inherits its geometric structure from $F P(n)$ and that the sectional curvature of these projective planes varies over [1,4] inclusive. $S^{n}(r)$ denotes as sphere of radius $r$ and curvature $1 / r^{2}$, while $\mathrm{Ca}(2)$ denotes the Cayley plane.

2. We are now in a position to state our main results. For simplicity, $S^{n}(1)$ will be denoted by $S^{n}$, otherwise we follow the conventions of the last section. See Remark 2.3 below.

THEOREM 2.1. The only nontrivial Riemannian foliations of $S^{n}$ by $S^{k}$ are the standard ones. In all cases the leaves are characterized as the fibers of a Riemannian submersion with totally geodesic fibers and hence the leaves are the fibers of a fiber bundle of one of the bundles below.
(a) $\begin{aligned} S^{1} \rightarrow & S^{3} \\ & \downarrow\end{aligned}$
$S^{2}\left(\frac{1}{2}\right)$
(b)
$S^{3} \rightarrow \underset{\downarrow}{S^{7}}$
$S^{4}\left(\frac{1}{2}\right)$
(c) $\begin{aligned} S^{7} \rightarrow & S^{15} \\ & \downarrow \\ & S^{8}\left(\frac{1}{2}\right)\end{aligned}$
(d)$$
S^{1} \rightarrow S^{2 n+1}
$$$$
C P(n), \quad n \geqslant 2,
$$

In fact, in classes (a), (d) or (e) any two such Riemannian submersions of the same class and the same total space $S^{m}$ differ by a fiber preserving isometry of $S^{m}$.

THEOREM 2.2. (a) The only nontrivial Riemannian foliations of $C P(n)$ by $C P(k)$ are those of $C P(2 n+1)$ by $C P(1)$. In this case, the leaf space is $H P(n)$ and the leaves of the foliation are characterized as the fibers of a Riemannian submersion with totally geodesic fibers and hence are the fibers of the fiber bundle

$$
\begin{aligned}
C P(1) & \rightarrow C P(2 n+1) \\
& \downarrow \\
& H P(n)
\end{aligned}
$$

If $n \geqslant 2$, any two such Riemannian submersions from $C P(2 n+1)$ differ by a fiber preserving isometry of the total space $C P(2 n+1)$.

(b) There are no nontrivial Riemannian foliations of $H P(n)$ by $H P(k)$ nor is there any Riemannian foliation of $\mathrm{Ca}(2)$ by $S^{8}=\mathrm{Ca}(1)$.

Proof of TheOREMS 2.1 AND 2.2. Except in the case of foliations of $S^{n}$ by $S^{1}$ all of the leaves are simply connected in 2.1 and 2.2 and hence they all have trivial foliation holonomy. We will show that for bundle-like foliations of $S^{n}$ by $S^{1}$ the foliation holonomy is trivial and hence the main result of (3) will apply to each of the foliations, since the leaves of the respective foliations are all compact manifolds. To show that the foliation holonomy of $S^{n}$ by $S^{1}$ is trivial observe that a result of Reinhart [8] implies that the holonomy of a Riemannian foliation with compact leaves is finite. In this case Lawson [7, p. 17] observes that the foliation in a saturated neighborhood $U$ of $S^{1}$ is equivalent to the foliation of the normal bundle 
(in this case the normal bundle of $S^{1}$ in $S^{n}$ ) given by its canonical flat linear connection. It suffices to show that the foliation of the normal bundle is a product. This will be the case if we can show that the normal bundle of $S^{1}$ in $S^{n}$ is trivial by Proposition 2.37 of [6], for example. Now $S^{1} \subset S^{n} \subset R^{n+1}$. Then the trivial normal bundle of $S^{1}$ in $R^{n+1}$ has the normal bundle of $S^{1}$ in $S^{n}$ as a direct summand. Hence, the normal bundle of $S^{1}$ in $S^{n}$ is trivial, since the normal bundle of $S^{n}$ in $R^{n+1}$ when restricted to $S^{1}$ is trivial.

Theorem 2.2 of [3] now applies to all the foliations admissible under the hypotheses of 2.1 and 2.2 above. Hence, each of these foliations can be described canonically as a Riemannian submersion from $M$ onto $B$, where $B$ is the leaf manifold. For Theorem 2.1 to follow it suffices to observe that since the leaves are spheres of radius 1 , they are connected and totally geodesic in $S^{n}$. The main result of [1, Theorem 3.5] applies and Theorem 2.1 follows.

To prove (a) of Theorem 2.2 observe that $C P(k)$ are the fibers of a Riemannian submersion $\pi: C P(n) \rightarrow B$, by [3]. Since the holomorphic curvature of $C P(k)$ is inherited from $C P(n)$, the Gauss equation implies that $C P(k)$ is totally geodesic in $C P(n)$. Since the foliation is now described as the fibers of a Riemannian submersion $\pi: C P(n) \rightarrow B$, an earlier result of the author in [2] shows that $\pi: C P(2 n+1)$ $\rightarrow H P(n)$ or possibly $\pi: C P(7) \rightarrow S^{8}\left(\frac{1}{2}\right)$. Ucci's paper [11] shows that, in fact, this second possibility cannot happen. Since for $n \geqslant 2$ any two Riemannian submersions $\pi_{1}$ and $\pi_{2}$ with $\pi_{i}: C P(2 n+1) \rightarrow H P(n)$ are equivalent by a fiber preserving isometry by the main result of [2]. Theorem $2.2(\mathrm{a})$ is proven completely.

We now sketch a proof of Theorem 2.2(b). As before such foliations arise from a Riemannian submersion $\pi: H P(n) \rightarrow B$ with leaves $H P(k)$ and, by [4], these leaves are totally geodesic. Composing $\pi$ with the natural Riemannian submersion $\pi_{1}$ : $C P(2 n+1) \rightarrow H P(n)$, we get a Riemannian submersion $\rho: C P(2 n+1) \rightarrow B$ with connected fibers. Using the work of [2], one can show that the leaves of $\rho$ are totally geodesic and invariant by the complex structure of $C P(2 n+1)$. But the only such Riemannian submersions occur when $C P(2 k+1)$ is the fiber of the composite submersion and $k=0$ by Theorem 2.2(a). Hence, the first part of Theorem 2.2(b) follows.

That no Riemannian foliation of $\mathrm{Ca}(2)$ by $S^{8}$ can occur is immediate from the main result of [3] and Serre's theorem on the Euler characteristic of a fibration.

REMARK 2.3. After this paper had been submitted, the author received preprints [12] and [13]. Ranjan's paper [13] significantly improves Theorem 3.5 of [1] using a different, intrinsic proof. Using Theorem A of this dissertation which he proves in Chapter 1 of [13], the last sentence of our Theorem 2.1 can be strengthened to read, "In fact, any two such Riemannian submersions of the same class and the same total space $S^{m}$ differ by a fiber preserving isometry of that $S^{m}$." With a little work this same result can be applied to Theorem 2.2(a) establishing the equivalence of any two such submersions from $C P(2 n+1) \rightarrow H P(n)$ for $n \geqslant 1$. See [13].

The main result of [12] recovers our Theorem 2.1 as a special case, using, however, a very different proof. I am grateful to the authors of [12] and [13] for providing me with copies of their beautiful papers. 
REMARK 2.4. Consider a Riemannian foliation of $S^{n}$ by $S^{1}$. In a recent letter Professor Gluck suggested an alternative approach to obtaining product coordinates in a neighborhood of $S^{1}$. Choose a transverse $(n-1)$ cell and from each point on it follows the great circle leaf through that point for a distance $\phi, 0 \leqslant \phi \leqslant 2 \pi$. This approach circumvents the appeal to general principles made in the beginning of the proof of Theorem 2.1.

ADDED IN PROOF. A significant generalization of Theorem 2.1 in the case when the leaf dimension is 1 has been obtained by Professors Gromoll and Grove and appears in the Rauch memorial volume in a paper entitled One dimensional metric foliations in constant curvature spaces.

\section{REFERENCES}

1. R. H. Escobales, Jr., Riemannian submersions with totally geodesic fibers, J. Differential Geom. 10 (1975), 253-276.

2. __ Riemannian submersions from complex projective space, J. Differential Geom. 13 (1978), 93-107.

3. ___ Sufficient conditions for a bundle-like foliation to admit a Riemannian submersion onto its leaf space, Proc. Amer. Math. Soc. 84 (1982), 280-284.

4. A. Gray, A note on manifolds whose holonomy group is a subgroup of $\mathrm{Sp}(n) \mathrm{Sp}(1)$, Michigan Math. J. 16 (1969), 125-128.

5. R. Hermann, A sufficient condition that a map of Riemannian manifolds be a fiber bundle, Proc. Amer. Math. Soc. 11 (1960), 236-242.

6. Walter A. Poor, Differential geometric structures, McGraw-Hill, New York, 1981.

7. H. Blaine Lawson, Jr., The quantitative theory of foliations, CBMS Regional Conf. Ser. in Math., no. 27, Amer. Math. Soc., Providence, R. I., 1977.

8. B. Reinhart, Closed metric foliations, Michigan Math. J. 8 (1961), 7-9.

9. R. T. Smith, The spherical representation of groups transitive on $S^{n}$, Indiana Univ. Math. J. 24 (1974), $307-325$.

10. __ Harmonic mappings of spheres, Amer. J. Math. 97 (1975), 364-385.

11. J. Ucci, On the nonexistence of Riemannian submersions from $C P(7)$ and $Q P(3)$, Proc. Amer. Math. Soc. 88 (1983), 698-700.

12. H. Gluck, F. Warner and W. Ziller, Fibrations of spheres by parallel great spheres, Univ. of Pennsylvania Preprint.

13. A. Ranjan, Riemannian submersions of Riemannian manifolds with connected totally geodesic fibres, Doctoral Thesis, Tata Inst. of Fundamental Research, Bombay, 1983.

Department of Mathematics, Canisius College, Buffalo, New York 14208 